\title{
Dynamic orthotic cranioplasty: treatment of the older infant.
}

\author{
Report of four cases
}

\author{
Timothy R. Littlefield, M.S., Jeanne K. Pomatto, B.O.C., And Kevin M. Kelly, Ph.D. \\ Cranial Technologies, Incorporated, Phoenix, Arizona, and Department of Occupational and \\ Environmental Health, College of Public Health, University of Iowa, Iowa City, Iowa
}

\begin{abstract}
The Dynamic Orthotic Cranioplasty Band is a cranial orthotic device used to treat deformational plagiocephaly in infants. The device works by applying a mild holding pressure to the most anterior and posterior prominences, where growth is not desired, while encouraging growth in adjacent flattened regions. Although this technique has been successfully used to treat infants as young as 3 months of age, it is often assumed that decreasing cranial growth and increasing cranial rigidity prohibit treatment in infants older than 1 year of age.

The authors' experience with older infants suggests the contrary. Through a series of case reports, they present evidence that this treatment remains viable during the 2 nd year of life and that improvement has been observed in infants in whom treatment has been initiated as late as 18 months. These examples certainly suggest that additional prospective studies are warranted.
\end{abstract}

KEY WORDS • deformational plagiocephaly - cranial orthosis • age

The term "deformational plagiocephaly" refers to a condition in which an infant's head becomes deformed because external forces were applied to the malleable cranium. This deformity may arise from prenatal as well as postnatal events. Prenatally, the deformity results from a restrictive intrauterine environment due to conditions such as a small maternal pelvis, abnormal uterine structure, large or multiple fetuses, paucity or excess of amniotic fluid, or increased maternal abdominal or uterine muscle tone..$^{3,14,20}$ Postnatally, cranial deformation has been attributed to a supine sleeping position,,$^{1,6,8,21}$ congenital muscular torticollis, ${ }^{4,7,9}$ neurological or cervical deficits, ${ }^{17}$ and premature birth. ${ }^{2,12}$

Our treatment protocol involves nonsurgical management in which a cranial orthotic device, the DOC band (Cranial Technology, Inc., Phoenix, AZ) is used. In this technique, a mild holding pressure is applied to the most anterior and posterior prominences of the cranium, where growth is not desired; at the same time, room is provided in the orthotic device to encourage growth in adjacent flattened regions. The efficacy of this technique in children treated before they reach 1 year of age has been presented in great detail by Levy, et al., (unpublished data) and others. ${ }^{10,13-15,19}$ Treatment options for older infants-those

Abbreviations used in this paper: DOC $=$ dynamic orthotic cranioplasty. presenting after 12 months of age-remain however, controversial.

It is generally believed that increasing cranial rigidity and decreasing cranial growth prohibit orthotic treatment in infants older than 1 year of age. However, as awareness of positional plagiocephaly has become more widespread and access to treatment more readily available, increasing numbers of older infants are presenting for evaluation. Although older infants still continue to represent only a small proportion of our treatment population, treatment results have been promising and, through a series of case reports, we present evidence suggesting that this treatment modality remains viable well into the 2 nd year of life.

\section{Case 1}

\section{CASE REPORTS}

This 18-month-old boy presented with severe positional plagiocephaly, which involved significant flattening of the right parietooccipital and left frontal regions, and an anteriorly positioned right ear. The infant was a singleton, delivered vaginally at full term, with a history of supine sleeping and neck tightness. The mother reported that the deformity was originally observed when the boy was 2 months of age, and the referring physician's notes stated: "Mother has known for a long period of time that the cranial vault was asymmetric but was assured that it would straighten on its own. She reports that it is no better than it was a year ago ...." 

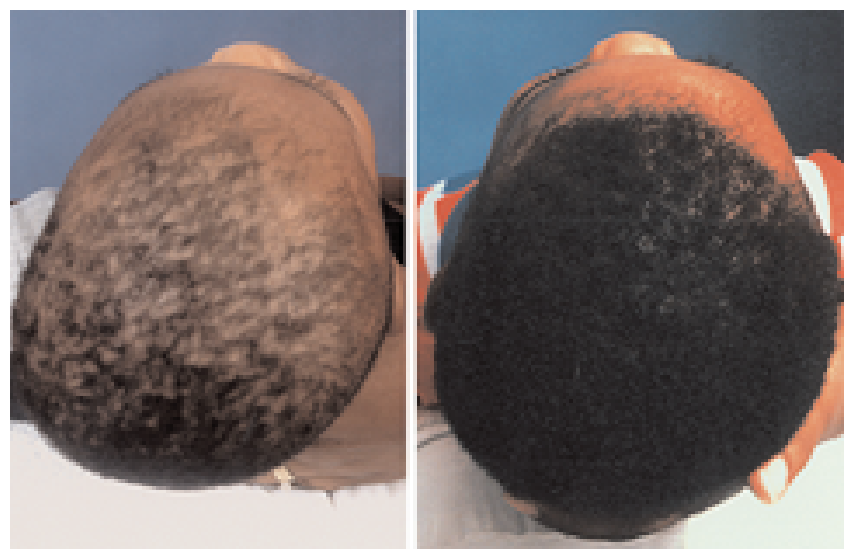

Fig. 1. Case 1. Left: Photograph showing the vertex of an 18month-old boy with right-sided plagiocephaly. Right: Posttreatment view of the vertex obtained after 5.5 months of DOC treatment.

When the infant began treatment at 18 months of age, there was $15 \mathrm{~mm}$ of cranial vault asymmetry, $7 \mathrm{~mm}$ of skull-base asymmetry, and $5 \mathrm{~mm}$ of upper-face asymmetry. After only 2 months of treatment, we observed a reduction in cranial vault asymmetry to $6 \mathrm{~mm}$. After 5.5 months of compliant treatment in which only a single orthotic device was required, cranial vault asymmetry had been reduced to $2 \mathrm{~mm}$, skull-base asymmetry to $1 \mathrm{~mm}$, and upper-face asymmetry to $3 \mathrm{~mm}$. During the same period, the patient's head circumference increased from 475 to $484 \mathrm{~mm}$ (Fig. 1).

\section{Case 2}

This 12-month-old boy was delivered vaginally at 36 weeks of gestational age. He had a history of supine sleeping and torticollis, causing him to favor turning his head to the right side. The patient's pediatrician first diagnosed the abnormal head shape when the infant was 2 months of age. The infant presented with significant flattening of the right parietooccipital region - greater right-sided posterior head height, and flattening of the left frontal region; the right ear was located anterior to the left ear. The patient entered treatment at 12 months of age. Treatment lasted 7.5 months and required two orthotic devices (Fig. 2).

\section{Case 3}

This 12-month-old male fraternal twin was delivered by Cesarean section at 33 weeks of gestational age. He presented with a history of in utero constraint and was reported to have remained in a transverse position throughout most of the pregnancy. The deformity was initially observed at birth and continued to worsen until he entered into treatment at 1 year of age, despite continual attempts to reposition the child off the flattened occiput. The infant presented with flattening of the right parietooccipital and left frontal regions; the right ear was located anterior to the left ear. Treatment began when the infant was 12 months of age and lasted for 4.5 months. The patient's parents reported that, by the end of treatment, he was beginning to remove the DOC band at his own discretion (Fig. 3).

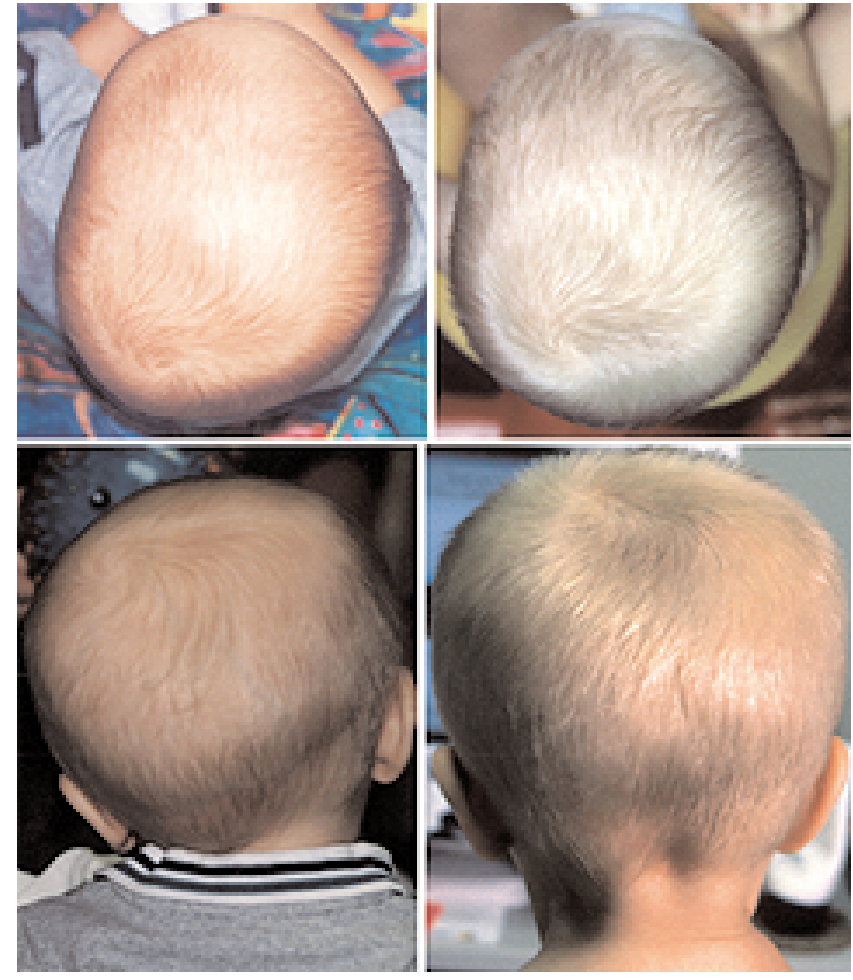

Fig. 2. Case 2. Pretreatment (upper left) and posttreatment (upper right) photographs showing the vertex of a 12-month-old boy with plagiocephaly and torticollis. Note the rounding of the right occiput and improvement in the frontal area after treatment. Pretreatment (lower left) and posttreatment (lower right) posterior views of the same infant. Neck-stretching exercises were instituted and, by the end of treatment, the infant demonstrates a near neutral position. Improvement in asymmetrical head height is also noted. Treatment lasted for 7.5 months and required two orthotic devices.

\section{Case 4}

This 20-month-old boy, also a fraternal twin, was delivered by Cesarean section at 39 weeks of gestational age. The abnormal head shape was initially noted at birth; however, the patient's parents were advised not to worry because the head shape would improve with time. The parents attempted to reposition the infant off the flattened occiput until he was 7 months of age. Still concerned that no improvement had been observed as anticipated, the mother consulted a specialist who, despite the patient's age (20 months) recommended DOC treatment due to the severity of the deformity. The infant presented for treatment with flattening of the left parietooccipital, right frontal regions; the left ear was located anterior to the right ear. The duration of treatment was 4.5 months, and only a single orthotic device was required (Fig. 4).

\section{DISCUSSION}

In this article, we have presented case examples demonstrating that orthotic treatment of deformational plagiocephaly may be extended into the 2nd year of life. Although a diminishing cranial growth curve and increasing cranial rigidity certainly present a greater challenge 


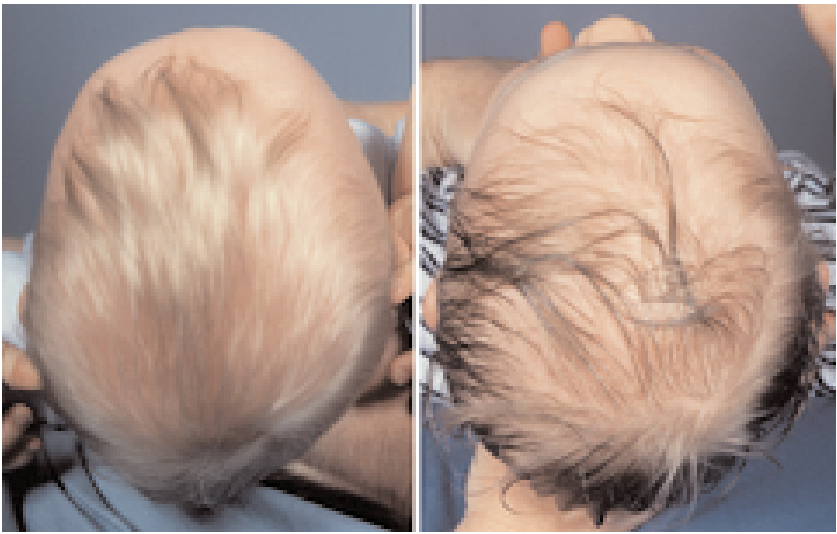

Fig. 3. Case 3. Pretreatment and (left) posttreatment (right) views of the vertex of a 12-month-old male fraternal twin (Baby A) with right-sided plagiocephaly. Treatment lasted 4.5 months and required a single orthotic device. Note the improvement in the overall configuration of the patient's head, as well as the right ear shift, which is often difficult to achieve after a patient reaches 12 months of age.

than that faced in treating younger patients, in our experience there is no reason to withhold treatment simply because the infant is approaching 1 year of age. Many infants in our program, who entered treatment at 9, 10, or 11 months of age, have greatly benefitted from treatment and continue to do well past their 1 st birthdays.

We believe that the ability to provide successful outcomes in these older infants is directly related to the design of the orthotic device and the treatment protocol followed. The dynamic technique was specifically designed as a more proactive approach, with each orthotic device custom made for the individual infant's deformity and pressures specifically applied to redirect symmetrical growth of the cranium. Initial improvement is commonly observed within the first several weeks, and the infant is examined on a weekly-to-biweekly basis so that adjustments to the orthotic device can be made to ensure that optimum correction is achieved. In our opinion, it is this
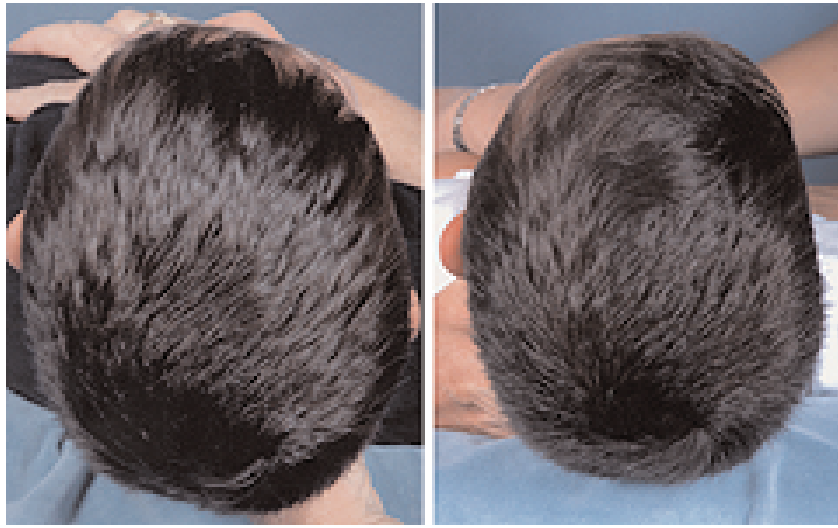

Fig. 4. Case 4. Pretreatment (left) and posttreatment (right) views of the vertex of a 20-month-old fraternal twin (Baby A) with left-sided plagiocephaly. Again, the overall configuration of the head is improved following 4.5 months of compliant treatment.
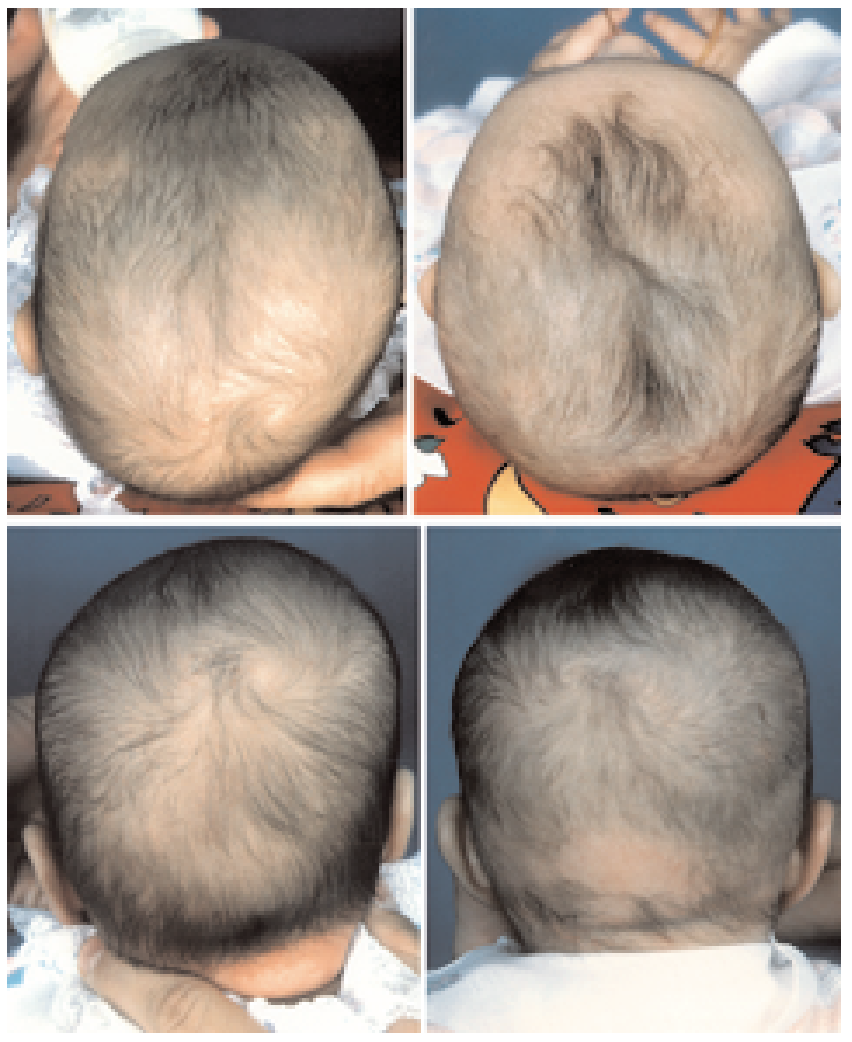

Fig. 5. Pretreatment (upper left) and posttreatment (upper right) views of the vertex of an infant treated for unilateral occipital plagiocephaly. Pretreatment (lower left) and posttreatment (lower right) posterior views of the same infant. Note the correction of the asymmetrical head height. Treatment lasted 5 weeks and began when the patient was 3.5 months of age. This rapid correction demonstrates the advantage of early intervention.

direct and continued involvement in the patient's care that has allowed us to expand the treatment window into the 2nd year of life because it more efficiently captures and redirects the remaining cranial growth.

Despite the fact that the DOC technique offers the opportunity to provide treatment to an infant older than 12 months of age, it is not our intention to suggest that treatment in younger infants should be delayed. In fact, we have long realized that the ideal candidate for orthotic treatment presents within the first 6 months of life (Fig. 5) and, in those patients who present at an older age, correction is typically slower and treatment takes longer. ${ }^{11}$ Unfortunately, delays in intervention caused by waiting for the head to "round out on its own" or by unsuccessful attempts at repositioning often push treatment beyond 12 months of age.

Clinical observations suggest that the untreated condition does not improve without intervention ${ }^{4,5,16,18,19,22}$ (Fig. 6). Likewise, we have found that repositioning is often attempted past an age when this would realistically be expected to be effective. More than $93 \%$ of the parents in our treatment program reported engaging in unsuccessful attempts to reposition their infants for a period of at least 2 months, with many indicating that repositioning was attempted for at least 4 to 6 months. Repositioning is most 

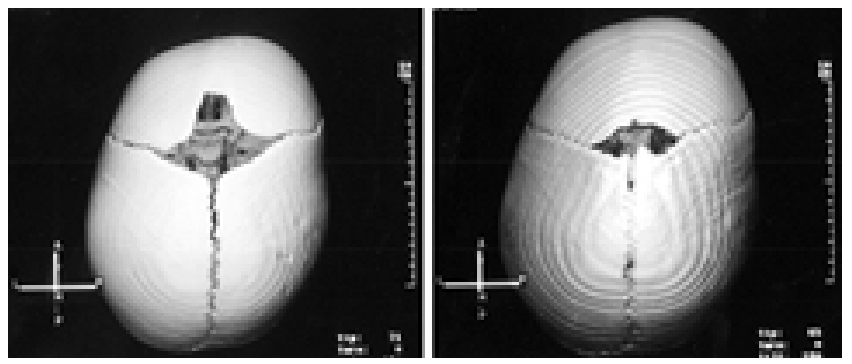

Fig. 6. Left: Three-dimensional computerized tomography scan obtained in a 7-month-old girl who was referred for cranial banding, but due to financial restraints could not undergo treatment. Physical therapy and a repositioning program were instituted. Right: At 19 months of age, 1 year later, it is noted that the skull is larger, but the deformity remains. (Images courtesy of Drs. David Matthews and Scott McLanahan, Charlotte, North Carolina.)

successful when it occurs before infants reach 4 months of age, after which time they have acquired the ability to reposition themselves. In the case of a moderate or severe deformity, repositioning may not be effective, regardless of age, because this would require the head to balance on the prominent aspect of the occiput, an inherently unstable position.

The authors acknowledge the limitations of the work presented, and recognize that additional prospective studies are warranted to present more definitive data demonstrating the efficacy of DOC treatment past 12 months of age. For infants who enter treatment after 1 year of age, it will be important to establish realistic expectations, because we certainly would not expect them to achieve the same level of correction observed when infants enter treatment at a much earlier age. ${ }^{11}$ Additionally, parents of older infants should be instructed to expect longer treatment times and, depending on the infants' specific growth patterns, a second orthotic device may be required to maximize the correction achieved. However, given the benefits of even a modest reduction in asymmetry, these are relatively minor concerns.

\section{DISCLOSURE}

Jeanne K. Pomatto is president of Cranial Technologies, Inc., and has a financial interest in the instrumentation advanced in this article. Mr. Littlefield is the Director of Research and Development at Cranial Technologies, Inc.; and Dr. Kelly is a paid consultant to that organization.

\section{References}

1. Argenta LC, David LR, Wilson JA, et al: An increase in infant cranial deformity with supine sleeping position. J Craniofac Surg 7:5-11, 1996

2. Baum JD, Searls D: Head shape and size of pre-term low-birthweight infants. Dev Med Child Neurol 3:576-581, 1971

3. Clarren SK, Smith DW: Congenital deformities. Pediatr Clin North Am 24:665-677, 1977

4. Clarren SK, Smith DW, Hanson JW: Helmet treatment for plagiocephaly and congenital muscular torticollis. J Pediatr 94: 43-46, 1979
T. R. Littlefield, J. K. Pomatto, and K. M. Kelly

5. Danby PM: Plagiocephaly in some 10-year old children. Arch Dis Child 37:500-504, 1962

6. Huang MH, Gruss JS, Clarren SK, et al: The differential diagnosis of posterior plagiocephaly: true lambdoid synostosis versus positional molding. Plast Reconstr Surg 98:765-776, 1996

7. Jones PG: Torticollis in Infancy and Childhood. Sternomastoid Fibrosis and the Sternomastoid "Tumor." Springfield, IL: Thomas, 1969

8. Kane AA, Mitchell LE, Craven KP, et al: Observations on a recent increase in plagiocephaly without synostosis. Pediatrics 97:877-885, 1996

9. Kawamoto HK: Torticollis versus plagiocephaly, in Marchac D (ed): Craniofacial Surgery. Proceedings of the First International Congress of the International Society of CranioMaxillo-Facial Surgery. New York: Springer-Verlag,1987, pp $105-109$

10. Kelly KM, Littlefield TR, Pomatto JK, et al: Cranial growth unrestricted during treatment of deformational plagiocephaly. Pediatr Neurosurg 30:93-199, 1999

11. Kelly KM, Littlefield TR, Pomatto JK, et al: Importance of early recognition and treatment of deformational plagiocephaly with orthotic cranioplasty. Cleft Palate Craniofac J 36: 127-130, 1999

12. Largo RH, Duc G: Head growth and changes in head configuration in healthy preterm and term infants during the first six months of life. Helv Paediatr Acta 32:431-442, 1978

13. Littlefield TR, Beals SP, Manwaring KH, et al: Treatment of craniofacial asymmetry with dynamic orthotic cranioplasty. J Craniofac Surg 9:11-19, 1998

14. Littlefield TR, Kelly KM, Pomatto JK, et al: Multiple-birth infants at higher risk for development of deformational plagiocephaly. Pediatrics 103:565-569, 1999

15. Littlefield TR, Pomatto JK, Beals SP, et al: Efficacy and stability of dynamic orthotic cranioplasty: an eight year investigation, in Whitaker LA (ed): Craniofacial Surgery 7. VIIth Biannual Meeting of the International Society of CranioMaxillo-Facial Surgery. Bologna: Monduzzi Editore, 1998, pp 109-111

16. Marsh JL, Vannier MW: Cranial deformities, in Marsh JL, Vannier MW, Stevens WG (eds): Comprehensive Care for Craniofacial Deformities. St. Louis: Mosby, 1985, pp 121-153

17. Moss M: The pathogenesis of artificial cranial deformation. Am J Phys Anthropol 16:269-286, 1958

18. Mulliken JB, Vander Woude DL, Hansen M, et al: Analysis of posterior plagiocephaly: deformational versus synostotic. Plast Reconstr Surg 103:371-380, 1999

19. Ripley CE, Pomatto J, Beals SP, et al: Treatment of positional plagiocephaly with dynamic orthotic cranioplasty. J Craniofac Surg 5:150-160, 1994

20. Smith DW, Graham JM: Smith's Recognizable Patterns of Human Deformation, ed 2. Philadelphia: WB Saunders, 1988

21. Turk AE, McCarthy JG, Thorne CHM, et al: The "back to sleep campaign" and deformational plagiocephaly: is there cause for concern? J Craniofac Surg 7:12-18, 1996

22. Watson GH: Relation between side of plagiocephaly, dislocation of hip, scoliosis, bat ears, and sternomastoid tumours. Arch Dis Child 46:203-210, 1971

Manuscript received July 17, 2000

Accepted in final form August 28, 2000.

Address reprint requests to: Timothy R. Littlefield, M.S., 1331 North 7th Street, Suite 170, Phoenix, Arizona 85006. email: littlefi @ cranialtech.com. 